\title{
Spatial damping of linear non-adiabatic magnetoacoustic waves in a prominence medium
}

\author{
M. Carbonell ${ }^{1}$, J. Terradas ${ }^{2}$, R. Oliver ${ }^{2}$, and J. L. Ballester ${ }^{2}$ \\ 1 Departament de Matemàtiques i Informàtica, Universitat de les Illes Balears, 07122 Palma de Mallorca, Spain \\ e-mail: marc. carbonell@uib.es \\ 2 Departament de Física, Universitat de les Illes Balears, 07122 Palma de Mallorca, Spain \\ e-mail: [dfsjlbo; jaume.terradas;ramon.oliver]@uib.es
}

Received 1 May 2006 / Accepted 19 July 2006

ABSTRACT

\begin{abstract}
Aims. We study the spatial damping of linear non-adiabatic magnetoacoustic waves in a homogeneous, isothermal, and unbounded medium permeated by a uniform magnetic field, with physical properties akin to those of solar prominences.

Methods. We consider an energy equation with optically thin radiative losses, thermal conduction, and heating, and linearize the MHD equations to obtain a sixth-order polynomial in the wavenumber $k$, which represents the dispersion relation for slow, fast, and thermal MHD waves. Since we are interested in the spatial damping, we have taken $\omega$ as real and have numerically solved the dispersion relation to obtain complex solutions for the wavenumber $k$ corresponding to fast, slow, and thermal waves.

Results. The thermal wave shows the strongest spatial damping, while the fast wave shows the weakest spatial damping. At periods greater than $1 \mathrm{~s}$ the spatial damping of magnetoacoustic waves is dominated by radiation, while at shorter periods the spatial damping is dominated by thermal conduction. For very short periods the isothermal regime is attained and the damping length becomes almost constant.

Conclusions. Radiative effects on linear magnetoacoustic slow waves can be a viable mechanism for the spatial damping of short period prominence oscillations, while thermal conduction does not play any role. In particular, short-period oscillations (5-15 min) observed in quiescent limb prominences, which seem to be due to internal fundamental slow modes, have damping lengths in the range $10^{4}-5 \times 10^{4} \mathrm{~km}$, in good agreement with our results.
\end{abstract}

Key words. Sun: oscillations - Sun: magnetic fields - Sun: corona - Sun: prominences

\section{Introduction}

Linear non-adiabatic waves can have interesting physical effects, such as time and spatial damping of disturbances, which are obtained by including different thermal mechanisms representing radiative losses and heating, as well as thermal conduction, in the energy equation. Following this approach, Ibañez \& Escalona (1993) studied the spatial damping and amplification of magnetosonic linear waves in an optically thin plasma with solar abundances in the temperature range $10^{4}-10^{8} \mathrm{~K}$. In their study, the Fourier analysis only includes the $k_{x}$ wavenumber, while among the thermal effects, perpendicular thermal conduction is included. To solve the problem, only the two extreme cases of propagation parallel and perpendicular to the magnetic field are considered, and the last one, which gives rise to a dispersion relation for coupled thermal and fast magnetoacoustic waves, is analysed in detail. Moreover, using non-adiabatic MHD waves, several studies have focused on the problem of coronal heating by proposing that heating can be provided by means of different mechanisms based on wave dissipation. For instance, Porter et al. (1994) made a short study of the damping length of MHD waves subject to dissipation by viscosity and thermal conduction in an unbounded magnetic plasma as a mechanism to heat the solar corona.

During recent years, thermal and non-thermal mechanisms have been proposed to explain the damping of linear longitudinal oscillations detected in coronal loops. For instance,
De Moortel et al. (2002) and De Moortel \& Hood (2003, 2004), using thermal conduction, compressive viscosity, gravitational stratification, and field line divergence, have studied the spatial damping of driven and non-driven slow MHD waves in coronal conditions, applying the obtained results to the case of standing and propagating slow waves in coronal loops observed with SOHO and TRACE. Also, Mendoza-Briceño et al. (2004) have performed numerical hydrodynamic calculations of a onedimensional loop model to investigate the effects of stratification on damping of longitudinal waves in the hot coronal loops observed by SUMER on board the SOHO satellite. In particular, they have studied the dissipation by thermal conduction and by compressive viscosity of standing slow magnetosonic disturbances in loops of semicircular shape.

Observational studies of small amplitude prominence oscillations have revealed a wide range of characteristic periods. The apparent tendency of periods to group below $10 \mathrm{~min}$ or in the range 40-80 min led to the distinction between shortand long-period oscillations. Later, more reports of periods in the range 10-40 min have been published and the intermediateperiod class emerged (Oliver \& Ballester 2002). It now appears well established that small amplitude, periodic changes in solar prominences do not normally affect the whole object at a time, but are of local nature instead. On the other hand, there are also some determinations of wavelength and phase speed obtained from observations of oscillations in slab-like prominences. For 
instance, Molowny-Horas et al. (1997) determined a maximum value $\lambda_{\|} \simeq 20000 \mathrm{~km}$, while the corresponding phase speed was $v_{\mathrm{p}} \leq 44 \mathrm{~km} \mathrm{~s}^{-1}$. Terradas et al. (2002) analysed small amplitude oscillations in a polar crown prominence reporting the presence, along two selected paths in the prominence region, of two plane propagating waves, as well as a standing wave. The plane waves propagate in opposite directions with wavelengths of 67500 and $50000 \mathrm{~km}$ and phase speeds of $15 \mathrm{~km} \mathrm{~s}^{-1}$ and $12 \mathrm{~km} \mathrm{~s}^{-1}$, while in the case of the standing wave, the estimated wavelength is of $44000 \mathrm{~km}$ and the phase speed of $12 \mathrm{~km} \mathrm{~s}^{-1}$. Also, Terradas et al. (2002) reported that the amplitude tends to decrease in time in such a way that the periodicity totally disappears after a few periods. Reliable values of the damping time, $\tau$, have been derived by Molowny-Horas et al. (1999) and Terradas et al. (2002) after fitting a sinusoidal function multiplied by a factor $\exp (-t / \tau)$ to different Doppler velocity time series. The values of $\tau$ thus obtained are usually between 1 and 3 times the corresponding period, and large regions of the prominence display similar damping times. Furthermore, the analysis made by Terradas et al. (2002) indicates that in the case of the propagating wave, which was interpreted as the slow mode, the amplitude of the oscillations spatially decreases in a substantial way in a distance of $2-5 \times 10^{4} \mathrm{~km}$ from the generation location. This distance can be considered as the typical spatial damping length, $L_{\mathrm{d}}$, of the oscillations.

Small amplitude oscillations in quiescent prominences have been interpreted in terms of MHD waves (Oliver \& Ballester 2002; Ballester 2006). When prominences are modelled as plasma sheets, short period oscillations (5-15 $\mathrm{min}$ ) observed in limb quiescent prominences seem to be produced by the internal fundamental slow modes of the prominence region, while long period oscillations (40-80 $\mathrm{min}$ ) are caused by the hybrid fundamental slow mode, which appears because of the coexistence of coronal and prominence media (Oliver \& Ballester 2002). Explanations for the time damping of prominence oscillations based on thermal mechanisms have been already proposed (Terradas et al. 2001; Terradas et al. 2005), while Ballai (2003) made a qualitative study of the spatial damping of linear compressional waves in solar prominences by considering different dissipative mechanisms such as isotropic and anisotropic viscosity, isotropic magnetic diffusivity, isotropic radiative damping (Newton's law), and anisotropic thermal conduction. He concludes that thermal radiation can explain the spatial damping of linear compressional waves and, also, that waves can damp due to anisotropic thermal conduction if they have short wavelengths. Then, keeping in mind the qualitative results derived in Ballai (2003) and the observational evidence that suggests that oscillations in prominences, and in the surrounding plasma, are of small amplitude, our aim here is to perform a complete quantitative study of the spatial damping of linear non-adiabatic magnetoacoustic waves in an unbounded medium with properties akin to those of solar prominences.

In Sect. 2, the equilibrium model, the linearised equations, and the general dispersion relation are presented. In Sect. 3 the general dispersion relation is solved and the results for the different regimes and heating mechanisms are shown. Finally, in Sect. 4, conclusions are drawn.

\section{Basic equations}

As a background model, we use a homogeneous unbounded medium threaded by a uniform magnetic field along the $x$-direction. The equilibrium magnitudes of the medium are given by

$p_{0}=$ const., $\rho_{0}=$ const., $T_{0}=$ const.,

$\boldsymbol{B}_{0}=B_{0} \hat{e}_{x}, \quad \boldsymbol{v}_{0}=0$

with $B_{0}=$ const. The effect of gravity has been ignored.

The basic MHD equations for the discussion of linear waves are:

$\frac{\mathrm{D} \rho}{\mathrm{D} t}+\rho \nabla \cdot v=0$

$\rho \frac{\mathrm{D} \boldsymbol{v}}{\mathrm{D} t}=-\nabla p+\frac{1}{\mu}(\nabla \times \boldsymbol{B}) \times \boldsymbol{B}+\rho g$,

$\rho T \frac{\mathrm{D} s}{\mathrm{D} t}+\rho L(\rho, T)-\nabla \cdot(\kappa \cdot \nabla T)=0$,

$\frac{\partial \boldsymbol{B}}{\partial t}=\nabla \times(\boldsymbol{v} \times \boldsymbol{B})$

$\nabla \cdot \boldsymbol{B}=0$,

$p=\frac{\rho R T}{\tilde{\mu}}$,

where $\frac{\mathrm{D}}{\mathrm{D} t}=\frac{\partial}{\partial t}+\boldsymbol{v} \cdot \nabla$ is the material derivative for time variations following the motion. In Eq. (3), the term $\nabla \cdot(\kappa \cdot \nabla T)$ represents the thermal conduction, although in our case perpendicular thermal conduction has been neglected, and $L$ is the heat-loss function that depends on the local plasma parameters. In the case of an equilibrium with uniform temperature, such as we consider here, the heat-loss function is

$L\left(\rho_{0}, T_{0}\right)=0$.

Usually, in solar applications this function represents the difference between an arbitrary heat input and a radiative loss function that, in our case, has been chosen as the optically thin radiative loss function (Hildner 1974). Then, our heat-loss function is given by

$L(\rho, T)=\chi^{*} \rho T^{\alpha}-h \rho^{a} T^{b}$,

where $\chi^{*}$ and $\alpha$ are piecewise functions depending on the temperature (Hildner 1974). The use of an optically thin plasma radiative cooling seems to be a reasonable approach for coronal, or almost coronal, conditions, while it may not be valid for prominence conditions because they are optically thick in some spectral lines. In this case, the radiative losses from the internal part of the prominence are greatly reduced and this can be represented by changing the exponent $\alpha$ in the cooling function, for temperatures $T \leq 10^{4} \mathrm{~K}$, from $\alpha=7.4$ to 17.4 (Milne et al. 1979) or $\alpha=30$ (Rosner et al. 1978), as well as by changing $\chi^{*}$ accordingly. Finally, the last term in Eq. (7) represents an arbitrary heating function that can be modified by taking different values for the exponents $a$ and $b$. In our case, different heating scenarios have been considered, and the values taken into account for the exponents $a$ and $b$ in Eq. (7) are (Rosner et al. 1978; Dahlburg \& Mariska 1988)

1. constant heating per unit volume $(a=b=0)$;

2. constant heating per unit mass $(a=1, b=0)$;

3 . heating by coronal current disipation $(a=1, b=1)$;

4. heating by Alfvén mode/mode conversion $(a=b=7 / 6)$; 
5. heating by Alfvén mode/anomalous conduction damping $(a=1 / 2, b=-1 / 2)$.

Considering small perturbations from the equilibrium in the form

$\boldsymbol{B}(t, \boldsymbol{r})=\boldsymbol{B}_{0}+\boldsymbol{B}_{1}(t, \boldsymbol{r}), p(t, \boldsymbol{r})=p_{0}+p_{1}(t, \boldsymbol{r})$,

$\rho(t, \boldsymbol{r})=\rho_{0}+\rho_{1}(t, \boldsymbol{r}), \quad T(t, \boldsymbol{r})=T_{0}+T_{1}(t, \boldsymbol{r})$,

$\boldsymbol{v}(t, \boldsymbol{r})=\boldsymbol{v}_{1}(t, \boldsymbol{r})$

we linearise the basic equations, (1)-(6), to obtain

$\frac{\partial \rho_{1}}{\partial t}+\rho_{0} \nabla \cdot \boldsymbol{v}=0$

$\rho_{0} \frac{\partial \boldsymbol{v}}{\partial t}=-\nabla p_{1}+\frac{1}{\mu}\left(\boldsymbol{B}_{0} \cdot \nabla\right) \boldsymbol{B}_{1}-\frac{1}{\mu} \nabla\left(\boldsymbol{B}_{0} \cdot \boldsymbol{B}_{1}\right)$,

$\frac{\partial p_{1}}{\partial t}-c_{\mathrm{s}}^{2} \frac{\partial \rho_{1}}{\partial t}=(\gamma-1)\left(\boldsymbol{B}_{0} \cdot \nabla\right)\left[\frac{\kappa_{\|}}{B_{0}^{2}}\left(\boldsymbol{B}_{0} \cdot \nabla\right) T_{1}\right]$ $-(\gamma-1) \rho_{0}\left(L_{\rho} \rho_{1}+L_{T} T_{1}\right)$,

$\frac{\partial \boldsymbol{B}_{1}}{\partial t}=\nabla \times\left(\boldsymbol{v} \times \boldsymbol{B}_{0}\right)$,

$\nabla \cdot \boldsymbol{B}_{1}=0$

$\frac{p_{1}}{p_{0}}-\frac{\rho_{1}}{\rho_{0}}-\frac{T_{1}}{T_{0}}=0$,

where $\kappa_{\|}=10^{-11} T^{5 / 2}$, and the factors $L_{\rho}, L_{T}$ are:

$L_{\rho}=\left(\frac{\partial L}{\partial \rho}\right)_{T}, L_{T}=\left(\frac{\partial L}{\partial T}\right)_{\rho}$,

with $T$ and $\rho$ held constant, respectively, at the equilibrium state. Now, since the medium is unbounded and uniform we can perform a Fourier analysis in plane waves and assume perturbations behaving like $\mathrm{e}^{\mathrm{i}(\omega t-k \cdot r)}$, and with no loss of generality, we choose the $z$-axis so that the wavevector $\boldsymbol{k}$ lies in the $x z$-plane, so that

$\boldsymbol{k}=k_{x} \hat{\boldsymbol{e}}_{x}+k_{z} \hat{\boldsymbol{e}}_{z}$.

Then, the following scalar equations are obtained

$\omega \rho_{1}-\rho_{0}\left(k_{x} v_{x}+k_{z} v_{z}\right)=0$,

$\omega \rho_{0} v_{x}-k_{x} p_{1}=0$,

$\omega \rho_{0} v_{y}+\frac{1}{\mu} B_{0 x} k_{x} B_{1 y}=0$,

$\omega \rho_{0} v_{z}-k_{z} p_{1}+\frac{1}{\mu} B_{0 x}\left(k_{x} B_{1 z}-k_{z} B_{1 x}\right)=0$,

$\omega B_{1 x}-B_{0 x} k_{z} v_{z}=0$,

$\omega B_{1 y}+B_{0 x} k_{x} v_{y}=0$

$\omega B_{1 z}+B_{0 x} k_{x} v_{z}=0$,

$\frac{p_{1}}{p_{0}}-\frac{\rho_{1}}{\rho_{0}}-\frac{T_{1}}{T_{0}}=0$,

$\mathrm{i} \omega p_{1}-\mathrm{i} \omega c_{\mathrm{s}}^{2} \rho_{1}+(\gamma-1)\left(\kappa_{\|} k_{x}^{2}+\rho_{0} L_{T}\right) T_{1}$

$+(\gamma-1) \rho_{0} L_{\rho} \rho_{1}=0$ where $c_{\mathrm{s}}=\sqrt{\frac{\gamma R T_{0}}{\tilde{\mu}}}$ is the sound speed. Equations (16) and (19) are decoupled from the rest and describe Alfvén waves, which are not investigated here. Next, we have eliminated the perturbations $p_{1}, T_{1}, \rho_{1}, B_{1 x}$, and $B_{1 z}$ in favour of $v_{x}$ and $v_{z}$, thus obtaining two algebraic equations for the velocity perturbations. Then, imposing that the determinant of this algebraic system be zero, factoring out the expression $-B_{0}^{2} k_{x}^{2}+\mu \omega^{2} \rho_{0}$, and taking $k_{x}=k \cos \theta$ and $k_{z}=k \sin \theta$, the resulting dispersion relation can be written as,

$b_{6} k^{6}+b_{4} k^{4}+b_{2} k^{2}+b_{0}=0$

with

$$
\begin{aligned}
b_{6}= & \frac{-\mathrm{i} c_{\mathrm{a}}^{2} T_{0}(\gamma-1) \kappa_{\|} \cos ^{4} \theta}{\rho_{0}}, \\
b_{4}= & c_{\mathrm{a}}^{2} c_{\mathrm{s}}^{2} \omega \cos ^{2} \theta+\mathrm{i}(\gamma-1)\left[c_{\mathrm{a}}^{2}\left(L_{\rho} \rho_{0}-L_{T} T_{0}\right) \cos ^{2} \theta\right] \\
& +\mathrm{i}(\gamma-1) \frac{T_{0} \kappa_{\|}\left(p_{0}+c_{\mathrm{a}}^{2} \rho_{0}\right) \omega^{2}}{p_{0} \rho_{0}} \cos ^{2} \theta, \\
b_{2}= & -\omega^{3}\left(c_{\mathrm{a}}^{2}+c_{\mathrm{s}}^{2}\right)-\mathrm{i} \omega^{2}(\gamma-1)\left(L_{\rho} \rho_{0}-L_{T} T_{0}\right) \\
& +\mathrm{i} \omega^{2}(\gamma-1) T_{0} \frac{2 c_{\mathrm{a}}^{2} L_{T} \rho_{0}-(1+\cos 2 \theta) \kappa_{\|} \omega^{2}}{2 p_{0}}, \\
b_{0}= & \frac{\omega^{4}\left(p_{0} \omega-(\gamma-1) \mathrm{i} L_{T} T_{0} \rho_{0}\right)}{p_{0}}
\end{aligned}
$$

where $c_{\mathrm{a}}=\frac{B_{0}}{\left(\mu \rho_{0}\right)^{0.5}}$ is the Alfvén speed.

A simple check of this general dispersion relation can be performed by setting $L_{T}=L_{\rho}=\kappa_{\|}=0$ in the coefficients of Eq. (23), so we obtain:

$\omega^{4}-\left(c_{\mathrm{a}}^{2}+c_{\mathrm{s}}^{2}\right) k^{2} \omega^{2}+c_{\mathrm{a}}^{2} c_{\mathrm{s}}^{2} k^{4} \cos ^{2} \theta=0$,

which is the well-known dispersion relation for adiabatic magnetoacoustic waves. Since we are interested in the spatial damping of magnetoacoustic waves we consider the frequency, $\omega$, to be real and seek complex solutions of the wavenumber $k$ expressed as $k=k_{\mathrm{r}}+\mathrm{i} k_{\mathrm{i}}$. Equation (23) is a sixth-order polynomial for $k$ as a function of $\omega$, and from it we can obtain three complex roots corresponding to the thermal, slow, and fast magnetoacoustic waves, respectively. In this case of spatial damping, an important difference with respect to the case of time damping is that the thermal wave is a propagating wave instead of a simple decaying oscillation. The wavelength of the waves is given by $\lambda=\frac{2 \pi}{k_{\mathrm{r}}}$, the damping length by $L_{\mathrm{d}}=\frac{1}{k_{\mathrm{i}}}$, and the damping length per wavelength is $\frac{L_{d}}{\lambda}$.

Considering typical values of the density, temperature, and magnetic field for prominences, we have explored prominence regimes with different radiative losses covering from optically thin to optically thick prominences. The different regimes considered imply that the values of the piecewise constants $\chi^{*}$ and $\alpha$, in the optically thin cooling function, must be modified (Table 1). For each of the considered prominence regimes, the five different heating mechanisms, listed before, have been taken into account.

\section{Results}

Now, for a magnetic field of $10 \mathrm{G}$ and a propagation angle of $\pi / 4$, the dispersion relation Eq. (23) has been solved numerically. Before considering the range of periods typical of the detected oscillations in prominences, we have solved Eq. (23) for prominence regime 1.1 , considering a period interval covering 
Table 1. Parameter values considered in the studied prominence regimes. All quantities are expressed in MKS units.

\begin{tabular}{ccccccc}
\hline \hline Regime & $T_{0}$ & $\rho_{0}$ & $\chi^{*}$ & $\alpha$ & $\tilde{\mu}$ & Reference \\
\hline Prominence (1.1) & 8000 & $5 \times 10^{-11}$ & $1.76 \times 10^{-13}$ & 7.4 & 0.8 & Hildner (1974) \\
Prominence (1.2) & 8000 & $5 \times 10^{-11}$ & $1.76 \times 10^{-53}$ & 17.4 & 0.8 & Milne et al. (1979) \\
Prominence (1.3) & 8000 & $5 \times 10^{-11}$ & $7.01 \times 10^{-104}$ & 30 & 0.8 & Rosner et al. (1978) \\
\hline
\end{tabular}
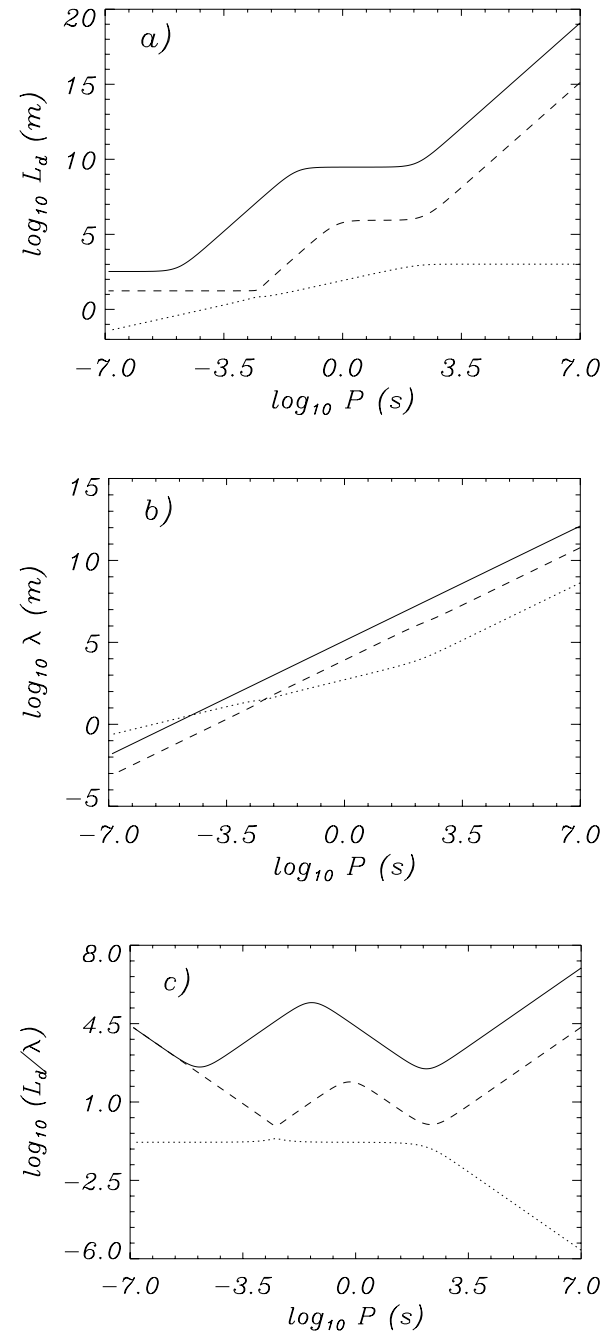

Fig. 1. Prominence regime 1.1 and a constant heating per unit volume $(a=b=0)$ : a) Damping versus period for fast (solid line), slow (dashed line), and thermal (dotted line) waves; b) wavelength versus period for fast (solid line), slow (dashed line), and thermal (dotted line) waves; c) damping length per wavelength for fast (solid line), slow (dashed), and thermal (dotted) waves.

from very short to very long periods. The behaviour of the damping length versus period for slow, fast, and thermal waves, in the case of constant heating per unit volume, is shown in Fig. 1a. This figure points out that, for any considered period, the thermal wave always has a shorter damping length than slow and fast waves, and that the damping lengths of magnetoacoustic waves increase with period. Figure $1 \mathrm{~b}$ shows the behaviour of the wavelength of the different waves with respect to the period, while Fig. 1c shows the damping length per wavelength for slow, fast, and thermal waves, pointing out the strong damping of the thermal wave. Now, and before proceeding further, we want to understand the behaviour of the damping length versus period for magnetoacoustic waves in Fig. 1. This understanding can be
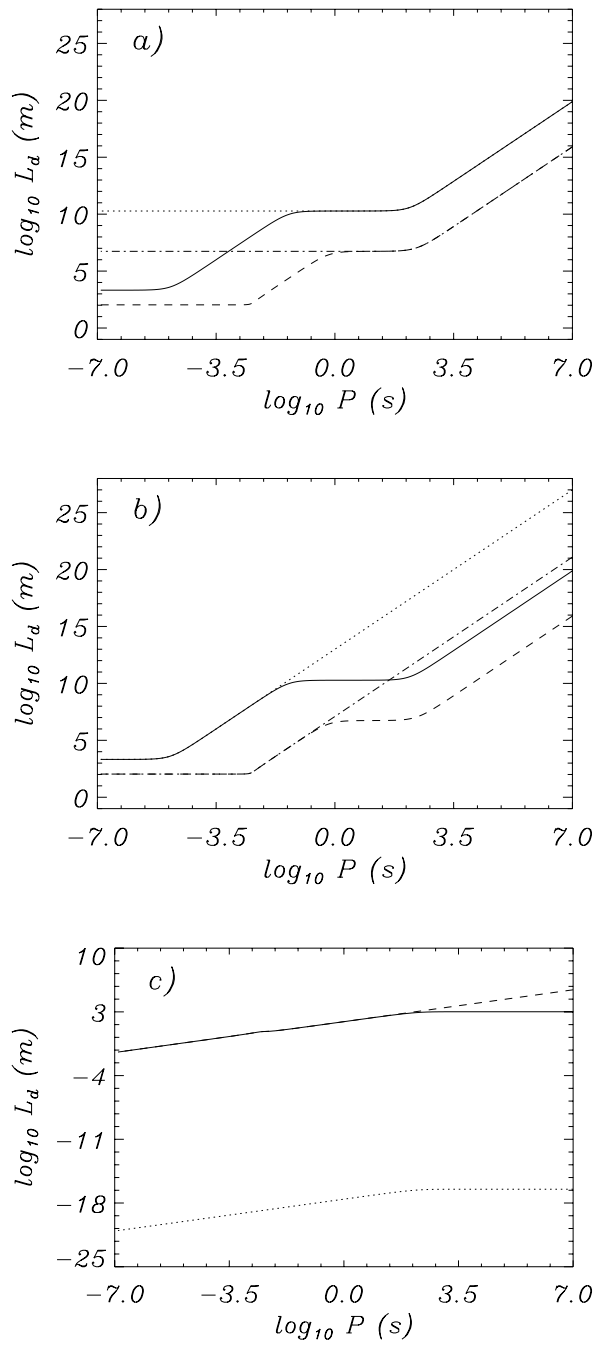

Fig. 2. Damping length versus period for prominence regime 1.1 and a constant heating per unit volume $(a=b=0)$. a) General case including radiation, heating, and thermal conduction: fast wave (solid line) and slow wave (dashed line); and the case including radiation, heating, and a negligible thermal conduction: fast wave (dotted line) and slow wave (dotted-dashed line); b) general case including radiation, heating, and thermal conduction: fast wave (solid line) and slow wave (dashed line); and the case including only thermal conduction: fast wave (dotted line) and slow wave (dotted-dashed line); c) thermal wave: general case including radiation, heating, and thermal conduction (solid line); case including only thermal conduction (dashed line); and case including radiation, heating, and a negligible thermal conduction (dotted line).

obtained by paying attention to Fig. 2. In Fig. 2a we display the damping lengths of magnetoacoustic waves for the general case, which includes radiation, heating, and thermal conduction, and for the case including radiation, heating, and a negligible thermal conduction, while in Fig. 2b the damping lengths for the general case, and the case including only thermal conduction are shown. A further case would be to remove the heating, but the results 


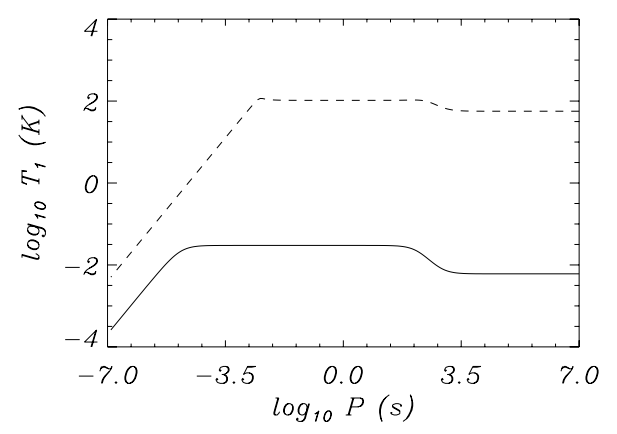

Fig. 3. Temperature perturbation versus period for slow (dashed line) and fast (solid line) waves for prominence regime 1.1 and a constant heating per unit volume $(a=b=0)$.

show no relevant difference with respect to the general case and this points out that the considered heating mechanisms have no strong influence on the behaviour of the damping lengths. The considered cases allow us to know when the different terms of the energy equation dominate the behaviour, with period, of the damping length of magnetoacoustic waves. Then, going from very long to very short periods we can see that the behaviour of the damping length is dominated by radiation for $P \geq 1 \mathrm{~s}$, while thermal conduction plays no important role (see Fig. 2a). However, once a certain value of the period $(P \approx 1 \mathrm{~s})$ is reached, thermal conduction starts to be important (see Fig. 2b) and the damping length suffers a new decrease as we move to smaller periods; finally, the last change in the behaviour of damping lengths, a new plateau, appears at very small periods and is due to the arrival to the isothermal regime. This arrival at the isothermal regime can be seen in Fig. 3, which shows the behaviour of the temperature perturbations with period. The largest perturbation corresponds to the slow wave and, comparing with Fig. 1, it can be seen that for both waves the transition to the low-period plateau of the damping length occurs exactly at the same period $\left(P \approx 10^{-2.5} \mathrm{~s}\right.$ and $P \approx 10^{-5} \mathrm{~s}$ for slow and fast waves, respectively) at which the temperature perturbation initiates a strong decrease due to the appearance of the isothermal regime. On the other hand, Fig. 2c shows the behaviour of the damping length versus period for the thermal wave for the general case, which includes radiation, heating, and thermal conduction, for the case including only radiation, heating and negligible thermal conduction, and for the case including only thermal conduction. In the figure it can be seen that at periods greater than $300 \mathrm{~s}$ the damping length is dominated by radiation, while at period below $300 \mathrm{~s}$, thermal conduction is dominant. Finally, when thermal conduction is made negligible, the damping length tends to zero because in the absence of thermal conduction the thermal wave disappears (see Eq. (30)). Finally, the strong spatial damping of the thermal wave pointed out by Figs. 1a and c suggests that this wave is quickly attenuated after its generation and, for this reason, in the following we will only consider the spatial damping of magnetoacoustic waves.

In the case of magnetoacoustic waves, the importance of radiation and thermal conduction can be also quantified in terms of two dimensionless parameters (De Moortel \& Hood 2004), namely, the thermal ratio,

$d=\frac{(\gamma-1) \kappa_{\|} T_{0} \rho_{0}}{\gamma^{2} p_{0}^{2} \tau_{\mathrm{s}}}=\frac{1}{\gamma} \frac{\tau_{\mathrm{s}}}{\tau_{\mathrm{cond}}}$, which is $1 / \gamma$ times the ratio of the sound travel time $\left(\tau_{\mathrm{s}}=l / c_{\mathrm{s}}\right)$ to the thermal conduction timescale $\left(\tau_{\text {cond }}=l^{2} p_{0} /\left[(\gamma-1) \kappa_{\|} T_{0}\right]\right)$, and the radiation ratio,

$r=\frac{(\gamma-1) \tau_{\mathrm{s}} \rho_{0}^{2} \chi^{*} T_{0}^{\alpha}}{\gamma p_{0}}=\frac{\tau_{\mathrm{s}}}{\tau_{\mathrm{r}}}$,

which is the ratio of the sound travel time to the radiation timescale $\left(\tau_{\mathrm{r}}=\gamma p_{0} /\left[(\gamma-1) \rho_{0}^{2} \chi^{*} T_{0}^{\alpha}\right]\right)$. From the equilibrium parameters, we can compute the value of $l$ at which the condition $d=r$ is satisfied,

$l=\left(\frac{\kappa_{\|}}{\rho_{0}^{2} \chi^{*} T_{0}^{\alpha-1}}\right)^{0.5}$

Then, when the spatial length of the perturbation, the wavelength, is of the order of $l$ or smaller, thermal conduction becomes dominant. Using the values assumed for prominence parameters, we obtain $l \approx 3700 \mathrm{~m}$, i.e., $\log l=3.56$. Figure $1 \mathrm{a}$ shows that, in the case of the slow wave, the dominance of thermal conduction appears, approximately, at $\log P=-0.4$, which corresponds, in Fig. $1 \mathrm{~b}$, to $\log \lambda=3.51$. In the case of the fast wave this dominance appears at $\log P=-1.04$, which corresponds to $\log \lambda=3.26$. Hence, both values of $\lambda$ are in close agreement with the found critical wavelength, $l$.

The end of the thermal conduction dominance and the beginning of the isothermal regime can be also quantified following Porter et al. (1994). For slow waves with $c_{\mathrm{s}}^{2} / c_{\mathrm{a}}^{2} \ll 1, \omega \approx c_{\mathrm{s}} k_{x}$ and the critical $\omega$ at which the transition to the isothermal regime starts is given by

$\omega_{\text {crit }}=\frac{2 n_{0} k_{\mathrm{B}} c_{\mathrm{s}}^{2}}{\kappa_{\|} \cos \theta}$.

A similar expression can be obtained for fast waves,

$\omega_{\text {crit }}=\frac{2 n_{0} k_{\mathrm{B}} c_{\mathrm{a}}^{2}}{\kappa_{\|} \cos ^{2} \theta}$.

In the case of slow waves, $\omega_{\text {crit }} \approx 2830 \mathrm{rad} / \mathrm{s}$, while for fast waves $\omega_{\text {crit }} \approx 460563 \mathrm{rad} / \mathrm{s}$, so $\log P_{\text {crit }}=-2.65$ for slow waves and $\log P_{\text {crit }}=-4.86$ for fast waves. Both values closely agree with the values of $\log P$ in Figs. 1a or 3 at which the beginning of the isothermal regime appears.

The periods that have been observed in small amplitude oscillations detected in quiescent prominences mostly vary between 1 and $90 \mathrm{~min}$. Then, within this range of periods, the spatial damping of the oscillations is driven by the radiative term, while thermal conduction plays no important role. A straightforward way to check this is to assume parallel propagation $(\theta=0)$. Then, Eq. (23) can be written as

$\left(\omega^{2}-c_{\mathrm{a}}^{2} k^{2}\right)\left\{\omega^{3}-\frac{\mathrm{i} T_{0}(\gamma-1)\left(k^{2} \kappa_{\|}+L_{T} \rho_{0}\right)}{p_{0}} \omega^{2}\right.$
$\left.-c_{\mathrm{s}}^{2} k^{2} \omega+\frac{\mathrm{i} k^{2}(\gamma-1)\left[k^{2} T_{0} \kappa_{\|}-\rho_{0}\left(L_{\rho} \rho_{0}-L_{T} T_{0}\right)\right]}{\rho_{0}}\right\}=0$.

The fast wave decouples from slow and thermal waves, which remain coupled, and propagates undamped. This behaviour is due to the fact that for field-aligned propagation, the fast wave becomes an Alfvén wave, characterised by not having density, pressure, and temperature perturbations, and therefore this 


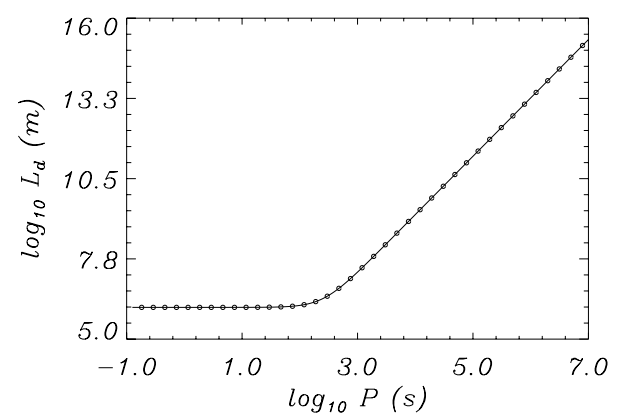

Fig. 4. Slow wave: damping length versus period obtained from the general dispersion relation given by Eq. (23) (solid line) and from the approximate analytical solution given by Eq. (31) (dotted line).

wave propagates undamped. Next, if we neglect thermal conduction, the remaining dispersion relation, corresponding to nonadiabatic slow waves, is given by

$$
\begin{aligned}
\omega^{3} & -\frac{\mathrm{i} T_{0}(\gamma-1) L_{T} \rho_{0}}{p_{0}} \omega^{2}-c_{\mathrm{s}}^{2} k^{2} \omega \\
& +\mathrm{i} k^{2}(\gamma-1)\left(L_{T} T_{0}-L_{\rho} \rho_{0}\right)=0
\end{aligned}
$$

which can be solved to obtain an analytical expression of the damping length versus frequency such as

$$
L_{\mathrm{d}}=\frac{\sqrt{2}}{\sqrt{\sqrt{\frac{\omega^{4}\left(c_{1}^{2}+\omega^{2}\right)}{c_{2}^{2}+c_{3}^{2} \omega^{2}}}-\frac{c_{1} c_{2} \omega^{2}-c_{3} \omega^{4}}{c_{2}^{2}+c_{3}^{2} \omega^{2}}}},
$$

with

$$
\begin{aligned}
& c_{1}=\frac{(\gamma-1) L_{T} T_{0} \rho_{0}}{p_{0}}, \\
& c_{2}=-(\gamma-1)\left(-L_{T} T_{0}+L_{\rho} \rho_{0}\right),
\end{aligned}
$$

and

$c_{3}=-c_{\mathrm{s}}^{2}$.

Figure 4 shows, in the case of parallel propagation, a comparison of the damping length of slow waves obtained from Eq. (23) and from the analytical solution given by Eq. (31). Both solutions display perfect agreement, fully confirming that for this range of periods the effect of thermal conduction is negligible.

\subsection{Exploring the parameter space}

In this section we modify the values of the physical parameters, such as magnetic field, temperature, and density, as well as different prominence regimes, the propagation angle, and the heating mechanism considered, in an attempt to know how the variation of all these parameters affects the damping length of MHD waves. Also, to note the interval of periods typically found in small amplitude oscillations of prominences, the white region in the corresponding figures defines the typical range of detected periods.

Figure 5 displays, for the different considered prominence regimes and a constant heating per unit volume, the damping length versus period for slow and fast waves. The interesting feature in these figures appears in the region where radiative losses dominate and this behaviour can be explained in terms of the critical wavelength, $l$ (Eq. (26)), which depends on $\chi^{*}$ and $\alpha$.
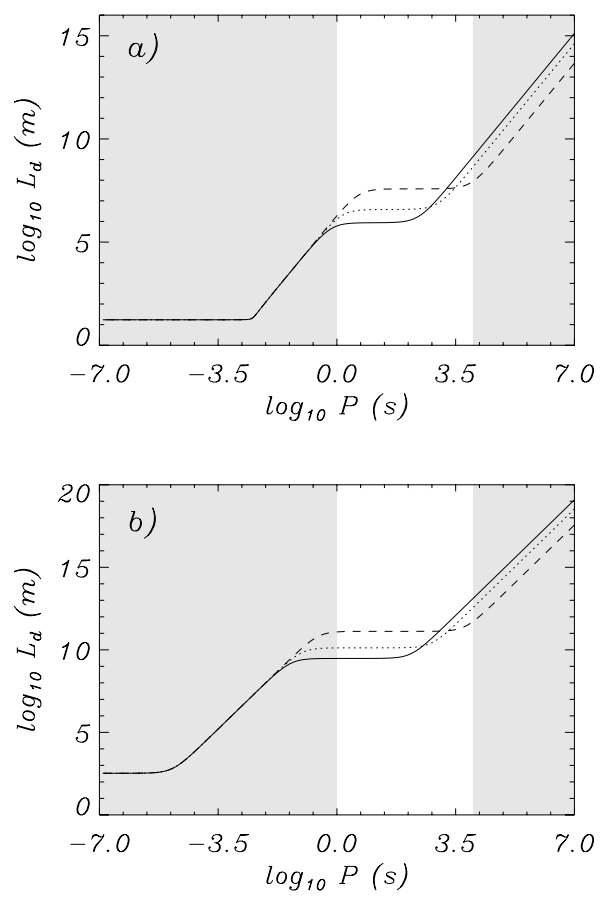

Fig. 5. Damping length versus period for a) slow and b) fast waves for a constant heating per unit volume $(a=b=0)$. The solid line corresponds to the prominence regime 1.1; the dotted line corresponds to the prominence regime 1.2; the dashed line corresponds to the prominence regime 1.3. The white region corresponds to the interval of periods typically found in prominences.

In regimes 1.2 and $1.3, \chi^{*}$ is smaller, while $\alpha$ is larger than in regime 1.1 , and the final result is that $l$ increases, which, according to Fig. 1b, corresponds to a larger period such as that seen in Figs. 5a, b. Also, in regimes 1.2 and 1.3, radiative losses decrease with respect to regime 1.1, and these two factors produce the rearrangement of the behaviour of the damping length in the region dominated by the radiative effects.

In the rest of the study we have only considered prominence regime 1.1 and have explored the influence of the magnetic field, temperature, density, propagation angle, and the heating mechanisms on the damping lengths of the magnetoacoustic waves.

Figure 6 displays the variation of the damping length when the magnetic field strength is modified. It shows that only the fast wave is affected by the variation of the magnetic field since the Alfvén velocity is modified. This change in the Alfvén velocity modifies the value of $\omega_{\text {crit }}$, so an increase (decrease) of the magnetic field means that $\omega_{\text {crit }}$ increases (decreases) and the arrival at the isothermal regime occurs at an smaller (larger) period; on the other hand, a change in the Alfvén velocity modifies several terms of the dispersion relation (23), which in turn modifies the behaviour of the damping length with period. Figure 7 displays the variation of the damping length when the temperature is modified and, in this case, several interesting features can be seen for both slow and fast waves. When the temperature of the prominence is increased, the damping length is smaller for short periods, but increases much more rapidly with the period; on the contrary, for lower temperatures the behaviour of the damping length with period is almost constant for short and intermediate periods and starts to increase for very long periods. All these behaviours can be understood by taking into account the fact that to modify the temperature means to modify the critical wavelength $l$ and the $\omega_{\text {crit }}$ for both waves, as well as the sound speed and the importance of radiative losses and thermal conduction. 

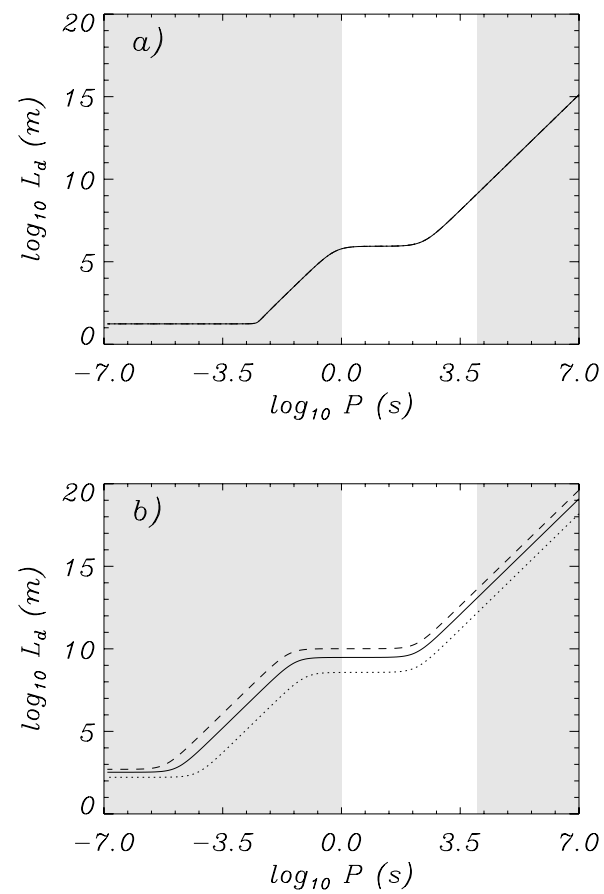

Fig. 6. Damping length versus period for a) slow and b) fast waves for a constant heating per unit volume $(a=b=0)$ for different values of the magnetic field strength. Prominence regime 1.1: the solid line $\left(B_{0}=1 \times 10^{-4} \mathrm{~T}\right)$, dotted line $\left(B_{0}=5 \times 10^{-4} \mathrm{~T}\right)$, and dashed line $\left(B_{0}=15 \times 10^{-4} \mathrm{~T}\right)$. The white region corresponds to the interval of periods typically found in prominences.

In fact, Fig. 7a shows that when the temperature is increased, the radiative plateau becomes smaller. Figure 8 displays the variation of the damping length when the density is modified. In this case, when we modify the density we modify $\omega_{\text {crit }}$, through $n_{0}$ and $l$, but radiative losses are also modified, as well as some terms in the dispersion relation (23). All these modifications give place to the complicated behaviour of the damping length versus period shown in Fig. 8.

The influence of the propagation angle has been also studied. Figure 9 displays the variation of the damping length of slow and fast waves when the propagation angle is changed. It can be seen that while the decrease of the angle does not substantially affect the damping length of slow waves, it strongly influences fast waves, increasing their damping length by a substantial amount. In the limit of parallel propagation, the fast wave propagates undamped since it becomes an Alfvén wave. Then, when the angle $\theta$ is decreased, going from perpendicular to parallel propagation, the damping length of fast waves increases rapidly becoming infinite for field-aligned propagation. On the contrary, when propagation is perpendicular to the magnetic field $(\theta=\pi / 2)$, Eq. (23) becomes a quadratic polynomial in $k$, and only a slightly damped, and thermally modified, fast wave and a thermal wave remain. Furthermore, in this case parallel thermal conduction plays no role and radiative losses are always dominant. Finally, Fig. 10 displays the variation of the damping length for slow and fast waves when the different heating mechanisms are considered, showing that the selected heating mechanism does not strongly affect the damping lengths of magnetoacoustic waves.
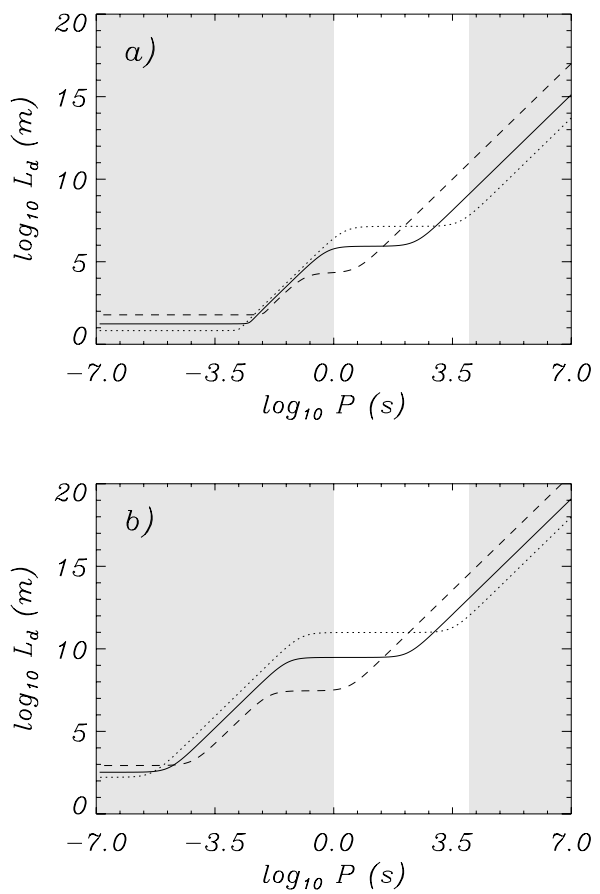

Fig. 7. Damping length versus period for a) slow and b) fast waves for a constant heating per unit volume $(a=b=0)$ for different values of the prominence temperature. The solid line $\left(T_{0}=8000 \mathrm{~K}\right)$, dotted line $\left(T_{0}=5000 \mathrm{~K}\right)$, and dashed line $\left(T_{0}=15000 \mathrm{~K}\right)$. The white region corresponds to the interval of periods typically found in prominences.

\section{Conclusions}

In this paper, our main aim has been to analyze the spatial damping of linear magnetoacoustic waves by the joint effect of optically thin radiation, thermal conduction, and a variety of heating terms. To this end, we have numerically solved the full dispersion relation for linear non-adiabatic MHD waves in an unbounded magnetised plasma and have studied the behaviour of the damping length of magnetoacoustic waves in a medium having physical properties akin to those of solar prominences.

The main conclusions that can be extracted from our study are:

1. Linear magnetoacoustic waves are spatially damped by thermal effects, and the strongest damping is obtained for slow waves.

2. For the case of perpendicular propagation, only damped fast and thermal waves propagate. On the contrary, for the case of parallel propagation, the fast wave decouples and propagates undamped, since it becomes an Alfvén wave, while the damped slow and thermal waves remain coupled. However, when termal conduction is absent, only a non-adiabatic slow wave remains.

3. The spatial damping of linear non-adiabatic magnetoacoustic waves is dominated by radiative effects or thermal conduction depending on the considered period. Imposing the condition that the thermal radio, $d$, be equal to the radiation ratio, $r$, a critical wavelength, $l$, can be obtained. Then, when the wavelength of the perturbation is smaller (greater) than $l$, thermal conduction (radiation) dominates. However, the dominance of thermal conduction ends when we arrive at the isothermal regime defined by a critical frequency $\left(\omega_{\text {crit }}\right)$. In the case of prominence regime 1.1 , considering only the slow wave and for the assumed typical values of density, temperature, and magnetic field in a quiescent prominence, 

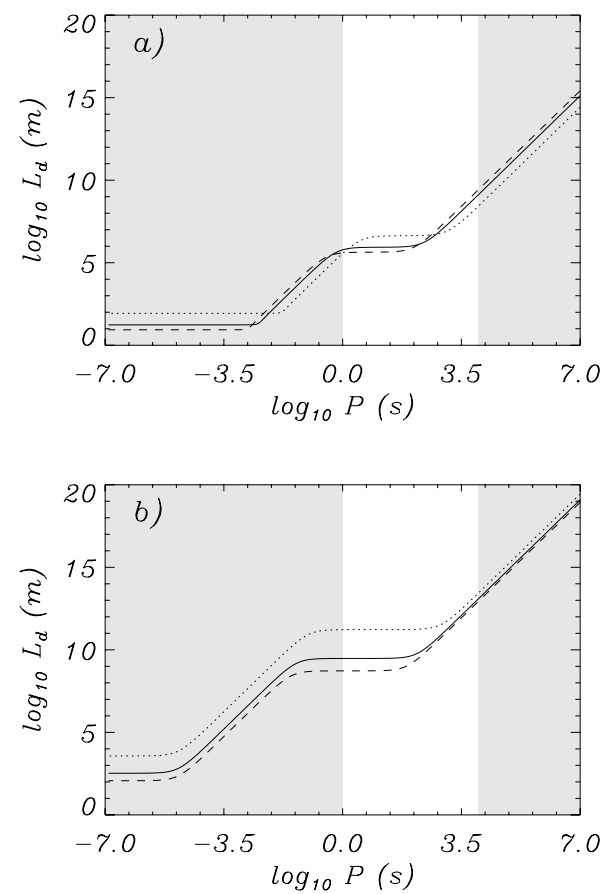

Fig. 8. Damping length versus period of a) slow and $\mathbf{b}$ ) fast waves for a constant heating per unit volume $(a=b=0)$ for different values of the prominence density. The solid line $\left(\rho_{0}=5 \times 10^{-11} \mathrm{~kg} \mathrm{~m}^{-3}\right)$, dotted line $\left(\rho_{0}=1 \times 10^{-11} \mathrm{~kg} \mathrm{~m}^{-3}\right)$, and dashed line $\left(\rho_{0}=10 \times 10^{-11} \mathrm{~kg} \mathrm{~m}^{-3}\right)$. The white region corresponds to the interval of periods typically found in prominences.

for periods greater than $1 \mathrm{~s}$ the damping is dominated by radiative effects; for periods between $10^{-2}$ and $1 \mathrm{~s}$ the damping is dominated by thermal conduction; finally, for periods smaller than $10^{-2} \mathrm{~s}$ we enter in the isothermal regime. A similar behaviour is found for the rest of the prominence regimes considered.

4. The behaviour of the damping length of the fast mode is similar to that of the slow mode, although its order of magnitude is larger.

5. The consideration of different prominence regimes, distinguished by the values of parameters $\alpha$ and $\chi^{*}$, introduces differences with respect to the value of the damping length. In particular, it can be seen that those values of the parameters $\alpha$ and $\chi^{*}$ that represent an optically thick prominence produce a substantial increment of the damping length for a certain range of periods. However, when very long periods are considered, the damping length is smaller than in the case of an optically thin prominence. This is due to the change in the value of the critical wavelength defining the change from the thermal conduction dominance regime to the radiation dominance regime.

6. Changes in density, temperature, and magnetic field, which affect the sound and Alfvén speeds, the critical wavelength, the critical frequency, and the radiative losses, modify the behaviour of the damping length of magnetoacoustic waves. This modification occurs in a complicated form and does not produce the same effect on the damping length at small, intermediate, or long periods.

7. The consideration of different heating mechanisms slightly affects the damping length of magnetoacoustic waves.

8. Taking into account the above results and the typical periods detected in small amplitude prominence oscillations, we can conclude that, for any considered prominence regime,
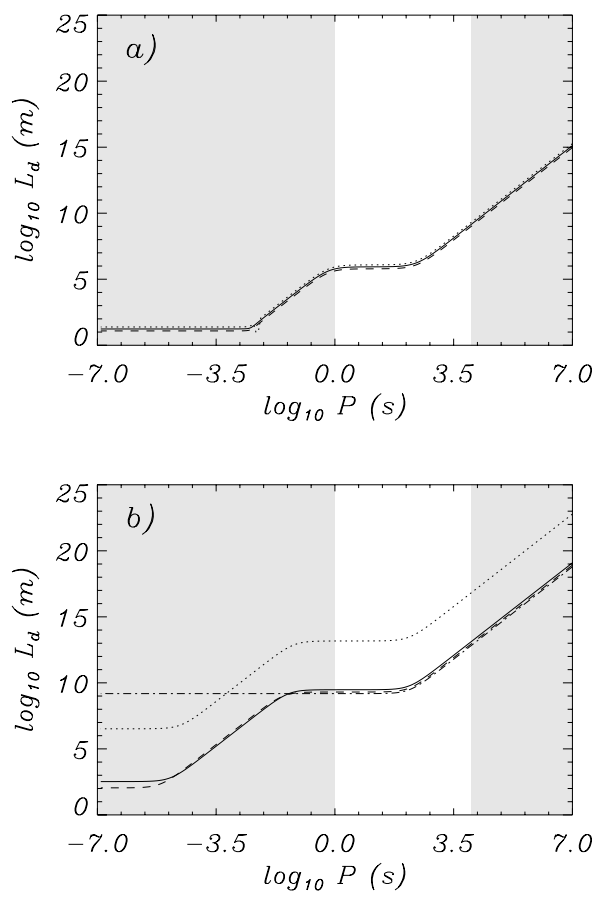

Fig. 9. Damping length versus period for the a) slow and b) fast waves for a constant heating per unit volume $(a=b=0)$ when the propagation angle is modified. The solid line $(\theta=\pi / 4)$, dotted line $\left(\theta=10^{-2}\right)$, and dashed line $(\theta=\pi / 3)$, dashed-dotted line $(\theta=\pi / 2)$. The white region corresponds to the interval of periods typically found in prominences.

the spatial damping of small amplitude prominence oscillations is dominated by radiative effects.

The influence of thermal conduction on the damping lengths of magnetoacoustic waves agrees with the suggestion made by Ballai (2003) in the sense that waves with very short wavelengths must be affected by damping due to thermal conduction. However, this dominance of thermal conduction is reduced to a range of wavelengths between $10^{-2} \mathrm{~km}$ and $10 \mathrm{~km}$ (see Figs. $1 \mathrm{~b}$ and $2 \mathrm{~b}$, together), since going to very short wavelengths, the arrival at an isothermal regime removes the influence of thermal conduction and the damping length becomes almost constant for both waves. Furthermore, as we have pointed out before, this effect of thermal conduction occurs in a range of periods that has not been observed yet in small amplitude prominence oscillations.

Although the model considered here is quite simple, it is interesting to compare the theoretical damping length with the value derived from the observations. In Terradas et al. (2002), the dominant period of the oscillations, interpreted as slow modes, was around $4500 \mathrm{~s}(75 \mathrm{~min})$ and the damping length was found to be in the range $1 \times 10^{7}-1 \times 10^{8} \mathrm{~m}$. Using this value of the period, we see from Figs. 5-10 that for the different regimes, the damping length for the slow mode is typically in the range $1 \times 10^{8}-1 \times 10^{9} \mathrm{~m}$. This value has a similar order of magnitude as the damping length estimated from the observations, which indicates that at least for this particular case, non-adiabatic MHD waves could be responsible for the spatial damping of the slow mode. Nevertheless, additional observations and more sophisticated theoretical models are needed to have a better comparison between observations and theory. Since we are only considering the prominence medium, we could estimate, for the interval of periods between $5 \mathrm{~min}$ and $15 \mathrm{~min}$, the damping length corresponding to slow wave 

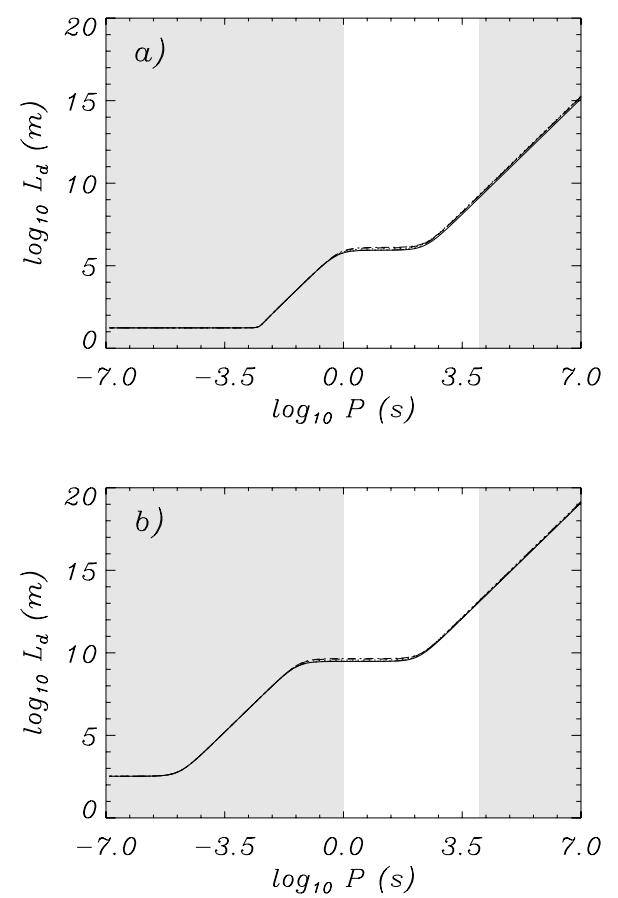

Fig. 10. Damping length versus period of the a) slow and $\mathbf{b})$ fast waves for the different heating mechanisms. The solid line $(a=b=0)$, dotted line $(a=1, b=0)$, dashed line $(a=b=1)$, dashed-dotted line $(a=$ $b=7 / 6)$, and dashed-double-dotted line $(a=1 / 2 ; b=-1 / 2)$. The white region corresponds to the interval of periods typically found in prominences.

which, in the case of prominence regime 1.1, varies between $10^{4} \mathrm{~km}$ and $5 \times 10^{4} \mathrm{~km}$ (see Fig. 5). For longer periods, the estimation of the damping length should take into account the influence of the corona where the dominant damping mechanism is thermal conduction. On the other hand, when the values of the physical parameters (density, temperature) characterising the prominence are modified, it seems difficult to estimate how the damping length of the slow waves will be modified. The reason is that to modify the density or the temperature implies a change in $\omega_{\text {crit }}$ and in the critical wavelength $l$, and these changes produce, mainly, a shift of the damping length versus period curves in the horizontal direction.

In summary, radiative effects on linear magnetoacoustic slow waves can be a viable mechanism for the spatial damping of short period prominence oscillations that seem to be characteristics of the prominence medium itself.

Acknowledgements. J. Terradas thanks the Spanish Ministry of Science and Education for the funding provided under a Juan de la Cierva fellowship. The authors acknowledge the financial support received from MCyT under grant AYA2003-00123.

\section{References}

Ballai, I. 2003, A\&A, 410, L17

Ballester, J. L. 2006, Proc. Phil. Trans. Roy. Soc. A, 364, 405

Dahlburg, R. B., \& Mariska, J. T. 1988, Sol. Phys., 117, 51

De Moortel, I., Hood, A. W., Ireland, J., \& Walsh, R. W. 2002, Sol. Phys., 209, 89

De Moortel, I., \& Hood, A. W. 2003, A\&A, 408, 755

De Moortel, I., \& Hood, A. W. 2004, A\&A, 415, 705

Hildner, E. 1974, Sol. Phys., 35, 123

Ibañez, M. H., \& Escalona, O. B. 1993, ApJ, 415, 335

Milne, A., Priest, E. R., \& Roberts, B. 1979, ApJ, 232, 304

Mendoza-Briceño, C. A., Erdélyi, R., \& Sigalotti, Leonardo di, G. 2004, ApJ, 605,493

Molowny-Horas, R., Oliver, R., Ballester, J. L., \& Baudin, F. 1997, Sol. Phys., 172,181

Molowny-Horas, R., Wiehr, E., Balthasar, H., Oliver, R., \& Ballester, J. L. 1999, in JOSO Annual Report 1998, ed. A. Antalová, H. Balthasar, \& A. Kucera, Astronomical Institute Tatranská Lomnica, 126

Oliver, R. J., \& Ballester, J. L. 2002, Sol. Phys., 206, 45

Porter, L. J., Klimchuk, J. A., \& Sturrock, P. A. 1994, ApJ, 435, 482

Rosner, R., Tucker, W. H., \& Vaiana, G. S. 1978, ApJ, 220, 643

Terradas, J., Oliver, R., \& Ballester, J. L. 2001, A\&A, 378, 635

Terradas, J., Molowny-Horas, R., Wiehr, E., et al. 2002, A\&A, 393, 637

Terradas, J., Carbonell, M., Oliver, R., \& Ballester, J. L. 2005, A\&A, 434, 741 